\title{
Application of Recycled Rubber from Scrap Tyres in the Adsorption of Toluene from Aqueous Solution
}

*11AMENAGHAWON, N.A; AISIEN, F.A; AGHO, O.E.

\author{
Department of Chemical Engineering, University of Benin \\ PMB 1154, Benin City, Nigeria \\ Phone: +234-806-9275563
}

KEYWORDS: Adsorption, Toluene, WTRG, adsorption capacity, Isotherm

\begin{abstract}
The adsorption of toluene from aqueous solution using waste tyre rubber granules (WTRG) was investigated in a batch system. The effect of various factors such as contact time, amount of adsorbent, size of adsorbent particles, and temperature of solution on the adsorption capacity of WTRG was determined. The equilibrium contact time was obtained to be 30 minutes indicating fast adsorption. The adsorption capacity of WTRG increased with decrease in particle size. Increasing the temperature of the aqueous solution resulted in a decrease in the adsorption capacity of WTRG while an increase in adsorbent dosage resulted in an increase in the adsorption capacity of WTRG. Kinetic and isotherm studies indicate that the adsorption of toluene was best described by the Lagergren pseudo-first order kinetic equation and Langmuir isotherm equation respectively. Results obtained indicate that recycled rubber from scrap tyres can be widely used as an adsorbent for the removal of toluene from aqueous solution. @ JASEM
\end{abstract}

\section{http://dx.doi.org/10.4314/jasem.v17i3.10}

The number of vehicles in Nigeria and consequently, the volume of traffic have increased over the years as a result of population increase, economic growth and expansion. This has led to an increase in the quantity of waste tyres generated annually in Nigeria. Aisien et al., (2006) recently reported that about 15 million scrap tyres are now in existence in Nigeria. These waste tyres represent a major environmental problem as most of them are discarded indiscriminately or stockpiled thereby attracting mosquitoes, rodents and some other dangerous reptiles (Mousavi et al., 2010). More so, fires resulting from tyre piles are nearly impossible to put out, release hazardous pollutants and are expensive clean up (Liu et al., 1998).

Although some of these waste tyres are recycled to obtain value added products such as tyre derived fuels, incorporation into asphalt for road pavement, floor mats, liquid waste treatment etc, a large quantity is still discarded improperly annually (Aisien et al., 2013). It is therefore imperative to search for alternatives to expand the re-use possibilities for waste tyres.

One of the methods employed to treat wastewater contaminated with hydrocarbons such as toluene, benzene, phenol and other aromatics is the utilization of materials such as activated carbon, ion exchange resins, zeolites and silicates (Mousavi et al., 2010. However, conventional adsorption using these materials is costly and its use is sometimes restricted on economic considerations.

Waste tyre rubber offers an excellent alternative to conventional adsorbents in that it is cheap and readily available. The use of waste tyre rubber as sorption media in wastewater treatment has been investigated. They have been used in various forms including waste tyre rubber granules, waste tyre rubber chips, waste tyre rubber ash etc. Kim et al., (1997) reported the sorption capacity of granulated waste tyre rubber for several substances. Smith et al., (2001) utilized rubber chips to treat water contaminated with phenol and p-cresol and it was determined that the rubber chips had a better uptake capacity for p-cresol than phenol.

The objective of this study was to examine the use of waste tyre rubber granules as an adsorbent for the removal of toluene from aqueous solutions. The study was focused on the sorption capability of waste tyre rubber for toluene from aqueous solution by testing the effect of various operational variables such as contact time, adsorbent dosage, adsorbent particle size, and temperature of the aqueous solution.

\section{MATERIALS AND METHODS}

Preparation of Adsorbent: Scrap tyres were provided by the Rubber Research Institute of Nigeria (RRIN) Iyanomo, Benin City, Nigeria. The tyres were washed with water to remove dirt and were subsequently air dried. The cleaned sides of the tyre free from steel breeds were cut into sections with the aid of a hacksaw and later into small pieces using very sharp knives. The size of the tyre chips were further reduced using an electric grinding machine. The resulting tyre particles were mechanically sieved to obtain particles in the size range 2.36 to $0.212 \mathrm{~mm}$ 
using different sieve trays. The tyre granules were then washed with distilled water to remove any foreign materials by agitating it with a mechanical shaker operating at $150 \mathrm{rpm}$ for 3 hours. It was subsequently oven dried at $60^{\circ} \mathrm{C}$ for 5 hours and stored in airtight containers for subsequent use (Alam et al., 2006). The surface structure and other properties of the WTRG were evaluated by nitrogen adsorption method at $-196^{\circ} \mathrm{C}$. Nitrogen adsorption isotherms were determined using an adsorption equipment (BET 624, Micro-meritics, Germany). The surface area of the WTRG was determined using the standard BET equation. Micropore volume was determined using the 3-D pore size distribution model developed at ISGS/UIUC while the total pore volume was determined at a relative pressure $(\mathrm{P} / \mathrm{Po})$ value of approximately 0.98 . The WTRG was also analyzed for carbon, nitrogen, oxygen, hydrogen, sulphur, moisture and ash contents using standard ASTM methods.

Preparation of Standard Solution: All chemicals used in this study were of analytical reagent grade and were used without further purification. Toluene (British Drug Houses Ltd, England) solution was prepared in a 1 liter amber glass bottle with a Teflonlined septum screw-on lid to minimize vaporisation. The appropriate amount of reagent grade toluene was extracted from its original container with a micro syringe and injected into the 1 liter bottle containing deionised water. The cap was then placed on the bottle and the solution was mechanically agitated for one minute. Standards solutions of volume $100 \mathrm{~mL}$ were prepared by adding a quantity of toluene in 250 $\mathrm{mL}$ amber bottles. Each bottle had a magnetic stirrer and was capped with a septum and sealed with paraffin.

Analysis of Toluene: The concentration of unadsorbed toluene in the sorption medium was measured using a UV-Vis spectrophotometer (PG Instruments model T70). A set of standard solutions of toluene were prepared and the absorbance of each standard solution was measured at a wavelength of $265 \mathrm{~nm}$. This value was obtained by scanning the UVVis spectrum of toluene and the wavelength that resulted in the maximum absorbance was selected.

Batch Adsorption Studies: Adsorption of toluene on dried waste tyre rubber granules was studied in batch experiments. The experiments were carried out in mechanically agitated stoppered $250 \mathrm{~mL}$ Erlenmeyer flasks. The batch study consisted of mixing a solute of a known concentration of toluene with water and crumb rubber. The solution was mechanically agitated until both the liquid and the solid medium reached chemical equilibrium at which point the toluene concentration on both solid and liquid medium remained constant. The adsorption capacity of the WTRG for toluene $q$ was expressed in terms of the amount of toluene retained by the WTRG. This is written as:
$q=\frac{V_{s}\left(C_{i}-C_{e}\right)}{W}$

Where: $\mathrm{q}=$ Equilibrium concentration of contaminant in the rubber $(\mathrm{mg} / \mathrm{g}), C_{i}=$ Initial concentration of contaminant in the solution $(\mathrm{mg} / \mathrm{L}), C_{e}=$ Equilibrium concentration of contaminant in the solution $(\mathrm{mg} / \mathrm{L})$, $V_{s}=$ Volume of solution $(\mathrm{L})$ and $W=$ Mass of crumb rubber $(\mathrm{g})$

\section{RESULTS AND DISCUSSION}

Characterisation of WTRG: The properties of waste tyre rubber granules as obtained from proximate analysis as determined by above tests methods are presented in Table 1. Ultimate elemental analysis of the WTRG indicate that the principal constituent was carbon accounting for about $87 \%$ of the weight of the granules followed by hydrogen which accounts for about $7 \%$ as shown in Table 2. The inorganic ash content of the WTRG used in this study obtained from ultimate analysis was about $3 \%$ as indicated in Table 2. The ash content is important because concentrations of trace species, especially metals, can likely affect the adsorptive properties of the WTRG. The values indicated in the Tables 1 and 2 are similar to those reported in the literature. The surface area, bulk density and pore volumes (micro and total) are indicated in Table 3.

Time dependent studies of the adsorption of toluene by WTRG: The profile of time dependent study of adsorption of toluene by WTRG is shown in Figure 1. It can be observed from the Figure that adsorption was rapid within the first 30 minutes as indicated by the steep increase in the adsorption capacity. The rate of adsorption levels off thereafter indicating that the adsorbent has been saturated and equilibrium reached. At equilibrium, all available active binding sites are occupied by the toluene molecules which causes some desorption to take place simultaneously with the adsorption process. As a result of this, no noticeable increase in adsorption of toluene is observed after the equilibrium contact time.

The equilibrium contact time of 30minutes indicates fast adsorption of toluene by the WTRG. The fast kinetic process observed can be attributed to the abundant availability of active binding sites on the adsorbent, which are later occupied as the adsorption process progresses, thereby resulting in the inability of the WTRG to remove toluene at later stages of the adsorption process (Mahvi et al., 2004). Gunasekara et al., (2000) reported fast adsorption (30 minutes) of naphthalene, toluene and mercury on granulated waste tyres. Alamo-Nole et al., (2006) reported an equilibrium contact time of 10 minutes for the sorption of ethylbenzene, toluene and xylene on granulated crumb tyre rubber.

Effect of adsorbent dosage on the adsorption of toluene on WTRG: Figure 2 shows the effect of adsorbent dosage on the adsorption capacity of WTRG for toluene. 
In general, it can be observed that the adsorption capacity increased with increase in adsorbent dosage indicating that adsorbent dose has a great influence on the adsorption process. It is evident from Figure 2 that increasing the adsorbent dose led to the enhancement of toluene uptake as a result of the increase in population (number) of active sites (Nagda et al., 2007). Maximum removal of toluene was observed with an adsorbent dose of $5 \mathrm{~g}$. According to Zafar et al., (2008), the amount of adsorbent added into the solution determines the number of binding sites available for adsorption. Alamo-Nole et al., (2006) reported maximum uptake capacity of crumb rubber for toluene at $\mathrm{pH} 1.5$ when $10 \mathrm{~g}$ of granulated crumb tyre rubber used. Mousavi et al., (2010) reported that increasing the adsorbent dose resulted in an increase in the adsorption capacity of the waste tyre rubber ash.

Effect of adsorbent particle size on the adsorption of toluene on WTRG: Figure 3 shows the adsorption capacity of WTRG for toluene at various sizes of adsorbent particles. The trend observed indicate that as the particle size is increased, adsorption capacity decreases. The maximum adsorption capacity of WTRG for toluene recorded was $18.18 \mathrm{mg} / \mathrm{g}$, obtained for a particle size of $0.212 \mathrm{~mm}$. The trend is explained by the fact that the smaller the size of the adsorbent particles, the greater the interior surface area and micro pore volume and consequently more active sites are available for adsorption (Annadurai et al., 2000). However, for larger particles, the pore diffusion resistance to mass transfer is higher and most of the internal surfaces of the particle may not be utilized for adsorption and consequently the amount of toluene adsorbed is small (Annadurai et al., 2000). Similar results were obtained by Rao and Bhole (2000) and Ajay et al., (2004).

Effect of solution temperature on the adsorption of toluene on WTRG: The effect of temperature on the WTRG/toluene system is shown in Figure 4. The adsorption capacity of WTRG generally decreased with increase in temperature from 5 to $45^{\circ} \mathrm{C}$. This indicates that a lower temperature is more favorable for the adsorption of toluene by WTRG. The trend observed is due to the weakening of the attractive force between the toluene molecules and the adsorbent on the one hand and due to enhancement of thermal energies of the adsorbate on the other hand thus making the attractive force between the adsorbate (toluene) and adsorbent insufficient to retain the adsorbed molecules at the binding sites (Jadhav and Vangara 2004).

Kinetics of adsorption: The kinetics of adsorption is important from the point of view that it controls the efficiency of the adsorption process. The Lagergren pseudo first-order, pseudo second-order and intra particle diffusion kinetic models were used to elucidate the mechanism of the adsorption process.

Lagergren pseudo first-order kinetic model: The
Lagergren rate equation is one of the most widely used kinetic equations for the adsorption of a solute from a liquid solution. The model assumes a first order adsorption kinetics and can be represented by the equation:

$$
\ln \left(q_{e}-q_{t}\right)=\ln q_{e}-k_{1} t
$$

Where: $q_{e}$ and $q_{t}$ are adsorption capacity at equilibrium and at time $t$, respectively $(\mathrm{mg} / \mathrm{g}), k_{l}$ is the rate constant of pseudo first order adsorption $\left(\min ^{-1}\right)$. The values of $\ln \left(q_{e}-q_{t}\right)$ were linearly correlated with $t$. The plot of $\ln \left(q_{e}-q_{t}\right)$ versus t should give a linear relationship from which $k_{l}$ and $q_{e}$ can be determined from the slop and intercept of the plot, respectively. Figure 5 depicts the pseudo-first order plot for the adsorption of toluene by WTRG. A linear relationship observed in the semi-log plot is indicative of the applicability of the above equation and the first order of the process. The first order rate constants calculated from the plots are given in Table 4.

Pseudo second-order kinetic model: The pseudosecond order kinetic model which is based on the assumption that chemisorption is the rate-determining step and can be expressed as in Equation (3):

$$
\frac{t}{q_{t}}=\frac{1}{h}+\frac{1}{q_{e}} t
$$

$$
\text { Where: } h=k_{2} q_{e}^{2}
$$

The plot of $\left(t / q_{t}\right)$ and $t$ of Equation (3) as presented in Figure 6 resulted in a linear relationship from which $q_{e}$ and $k_{2}$ can be determined from the slope and intercept of the plot, respectively. The respective constant values are given in Table 4.

Intra particle diffusion model: The intra particle diffusion kinetic model can be written as presented in Equation (4):

$$
q_{t}=K_{p} t^{1 / 2}+C
$$

Where $K p$ is the intra particle diffusion rate constant $\left(\mathrm{mgg}^{-1} \mathrm{~min}^{-1 / 2}\right)$ and $C$ is the boundary layer thickness.

The intercept of the plot reflects the boundary layer effect. The larger the intercept, the greater is the contribution of the surface sorption in the rate controlling step. The result presented in Figure 7 indicates the existence of some boundary layer effect and further showed that intra particle diffusion was not the only rate limiting step. The calculated diffusion coefficient values are presented in Table 4.

Generally, all the tested adsorption kinetic models fitted well to the adsorption kinetic data with high correlation coefficients; however, the Lagergren firstorder gave the best fit with the highest correlation coefficient to describe the adsorption behaviour of toluene onto WTRG.

Isotherm Studies: To examine the relationship 
between toluene uptake $\left(\mathrm{q}_{\mathrm{e}}\right)$ and its equilibrium concentration in the solution $\left(\mathrm{C}_{\mathrm{e}}\right)$, adsorption isotherm models are widely employed for fitting experimental data, of which the Langmuir and Freundlich isotherm equations are the most widely used. The curves of the related adsorption isotherms are regressed and parameters of the equation are thus obtained.

Langmuir Isotherm: The Langmuir model has been used empirically because it contains the two useful parameters $\left(q_{o}\right.$ and $\left.b\right)$, which reflect the two important characteristics of the sorption system. It provides information on uptake capabilities and is capable of reflecting the usual equilibrium adsorption process behavior. The linear form of the Langmuir equation is given as:

$$
\frac{C_{e}}{q_{e}}=\frac{1}{b q_{o}}+\frac{C_{e}}{q_{o}}
$$

$\mathrm{q}_{\mathrm{o}}$ is the maximum sorption capacity $(\mathrm{mg} / \mathrm{g})$ of the adsorbent while $b$ is the sorption constant $(\mathrm{L} / \mathrm{mg})$ at a given temperature. A linear plot of $\mathrm{C}_{\mathrm{e}} / \mathrm{q}_{\mathrm{e}}$ against $\mathrm{C}_{\mathrm{e}}$ as shown in Figure 8 was employed to obtain the values of $\mathrm{q}_{\mathrm{o}}$ and $\mathrm{b}$ from the slope and intercept of the plot respectively.

The values of the Langmuir isotherm parameters as well as the correlation coefficient $\left(R^{2}\right)$ of the Langmuir equation for the adsorption of toluene by WTRG are given in Table 5.

Freundlich Isotherm: The Freundlich isotherm is an empirical equation employed to describe heterogeneous systems. The Freundlich equation is expressed as:

$$
q_{e}=K_{f}\left(C_{e}\right)^{1 / n}
$$

This equation can be expressed in linear form as follows:

$$
\operatorname{lnq}_{e}=\ln K_{f}+1 / n \ln C_{e}
$$

$\mathrm{K}_{\mathrm{f}}$ and $\mathrm{n}$ are the Freundlich constants related to the adsorption capacity and adsorption intensity respectively. The intercept and slope of the linear plot of $\ln \mathrm{q}_{\mathrm{e}}$ against $\ln \mathrm{C}_{\mathrm{e}}$ at given experimental conditions as shown in Figure 10 provides the values of $K_{f}$ and $n$. Values of $n$ between 1 and 10 represent beneficial adsorption. The values of these parameters as well as the correlation coefficient $\left(\mathrm{R}^{2}\right)$ of the Freundlich equation for the adsorption of toluene by WTRG are given in Table 5.

Results presented in Table 5 indicate that the experimental data fitted the Langmuir isotherm better than the Freundlich isotherm as evident in the higher $\mathrm{R}^{2}$ value obtained for the Langmuir isotherm. This suggests that the adsorption of toluene by WTRG is of the mono-layer type.

Conclusions The present study investigated the adsorption of toluene from aqueous solution using waste tyre rubber granules in a batch system. The following conclusions can be drawn.

The equilibrium contact time was obtained as 30 minutes indicating that the adsorption process was a fast kinetic process.

A low temperature $\left(5^{\circ} \mathrm{C}\right)$, small adsorbent particle size $(0.212 \mathrm{~mm})$ and higher adsorbent dosage favoured the adsorption process with removal capacities of 17.57, 18.18 and $27.83 \mathrm{mg} / \mathrm{g}$ respectively.

Kinetic and isotherm studies indicate that the adsorption of toluene was best described by the Lagergren pseudo-first order kinetic equation and Langmuir isotherm equations respectively.

This study has demonstrated that the low cost WTRG can be widely used for removal of toluene from aqueous solution.

Table 1: Properties of WTRG obtained from proximate analysis

\begin{tabular}{|c|c|c|c|}
\hline \multirow{2}{*}{ Property } & \multicolumn{3}{|c|}{ Value (wt\%) } \\
\cline { 2 - 4 } & $\begin{array}{c}\text { This } \\
\text { work }\end{array}$ & $\begin{array}{c}\text { Lee et al., } \\
(1995)\end{array}$ & $\begin{array}{c}\text { Gonzalez et al., } \\
(2001)\end{array}$ \\
\hline Fixed carbon & 28.35 & 28.50 & 29.20 \\
\hline Moisture & 0.51 & 0.50 & 0.70 \\
\hline Ash & 7.60 & 3.7 & 8.0 \\
\hline Volatile & 63.54 & 67.30 & 61.90 \\
\hline
\end{tabular}

Table 2: Ultimate elemental analysis of WTRG

\begin{tabular}{|c|c|c|c|}
\hline Property & This work & $\begin{array}{c}\text { Cunliffe and } \\
\text { Williams } \\
(1998)\end{array}$ & $\begin{array}{c}\text { Gonzales et al., } \\
(2001)\end{array}$ \\
\hline Carbon & 86.50 & 86.4 & 86.7 \\
\hline Hydrogen & 6.64 & 8.0 & 8.1 \\
\hline Oxygen & 1.10 & 3.4 & 1.3 \\
\hline Nitrogen & 0.40 & 0.5 & 0.4 \\
\hline Sulphur & 2.0 & 1.7 & 1.4 \\
\hline Inorganic Ash & 2.85 & 2.4 & 2.9 \\
\hline
\end{tabular}

\section{${ }^{* 1}$ AMENAGHAWON, N.A; AISIEN, F.A; AGHO, O.E.}


Table 3: Physical properties of WTRG

\begin{tabular}{|c|c|}
\hline Property & Value \\
\hline $\begin{array}{c}\text { Surface area } \\
\left(\mathrm{m}^{2} / \mathrm{g}\right)\end{array}$ & 0.20 \\
\hline Bulk density $\left(\mathrm{g} / \mathrm{cm}^{3}\right)$ & 0.23 \\
\hline Total pore volume $\left(\mathrm{cm}^{3} / \mathrm{g}\right)$ & 0.66 \\
\hline Micro pore volume $\left(\mathrm{cm}^{3} / \mathrm{g}\right)$ & 0.23 \\
\hline
\end{tabular}

Table 4: Parameter values for pseudo first order, pseudo second order and intraparticle diffusion models

\begin{tabular}{|c|c|c|}
\hline $\begin{array}{c}\text { Adsorption Kinetic } \\
\text { Model }\end{array}$ & Parameters & Values \\
\hline \multirow{2}{*}{$\begin{array}{c}\text { Lagergren Pseudo } \\
\text { First-Order }\end{array}$} & $k_{l}\left(\mathrm{~min}^{-1}\right)$ & 0.059 \\
\cline { 2 - 3 } & $q_{e}(\mathrm{mg} / \mathrm{g})$ & 22.09 \\
\cline { 2 - 3 } & $R^{2}$ & 0.99 \\
\hline \multirow{3}{*}{ Pseudo Second-Order } & $k_{2}\left(\mathrm{gmg}^{1} \mathrm{~min}^{-1}\right)$ & 0.002 \\
\cline { 2 - 3 } & $q_{e}(\mathrm{mg} / \mathrm{g})$ & 27.03 \\
\cline { 2 - 3 } Intra particle diffusion & $R^{2}$ & 0.96 \\
\cline { 2 - 3 } & $K_{p}$ & 2.45 \\
\cline { 2 - 3 } & $C$ & 0.38 \\
\hline
\end{tabular}

Table 5: Kinetic parameters for Langmuir and Freundlich

$$
\text { isotherms }
$$

\begin{tabular}{|c|c|c|c|c|c|}
\hline \multicolumn{3}{|c|}{ Langmuir isotherm } & \multicolumn{2}{c|}{ Freundlich isotherm } \\
\hline $\mathrm{q}_{\mathrm{o}}(\mathrm{mg} / \mathrm{g})$ & $\begin{array}{c}\mathrm{b} \\
(\mathrm{L} / \mathrm{mg})\end{array}$ & $\mathrm{R}^{2}$ & $\begin{array}{c}\mathrm{K}_{\mathrm{f}} \\
(\mathrm{mg} / \mathrm{g})\end{array}$ & $\mathrm{n}$ & $\mathrm{R}^{2}$ \\
\hline 200 & 0.008 & 0.99 & 0.039 & 1.06 & 0.95 \\
\hline
\end{tabular}

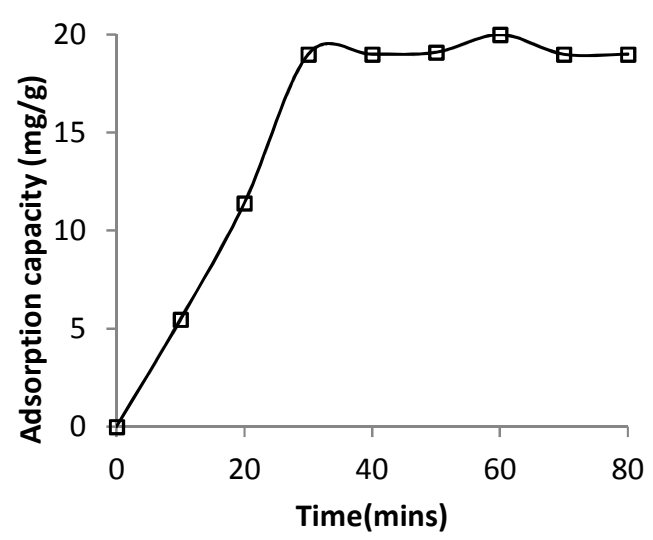

Fig. 1: Effect of contact time on the adsorption capacity of WTRG (pH 8.5; initial concentration, $500 \mathrm{mg} / \mathrm{L}$; WTRG dose, $2 \mathrm{~g}$; temperature, $20^{\circ} \mathrm{C}$ ).

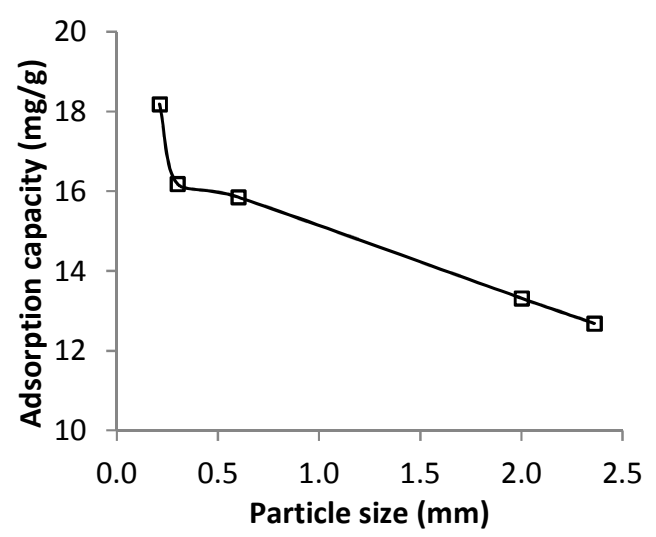

Fig. 3: Effect of adsorbent particle size on the adsorption of toluene by WTRG ( $\mathrm{pH} 8.5$; WTRG dose, $2 \mathrm{~g}$; initial concentration, $500 \mathrm{mg} / \mathrm{L}$; temperature, $20^{\circ} \mathrm{C}$ ).

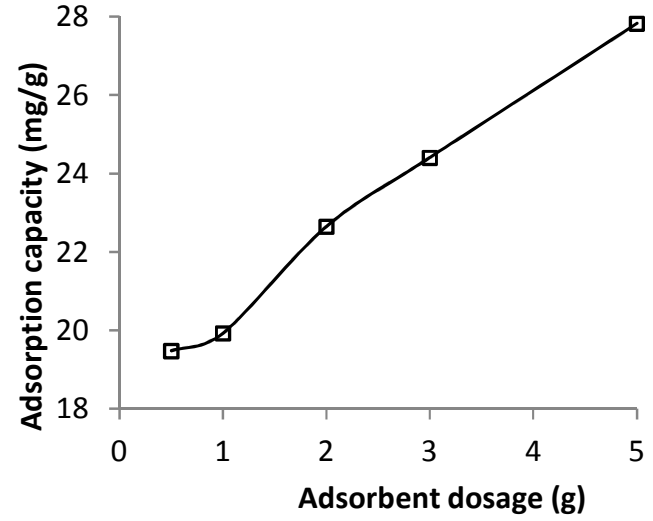

Fig. 2: Effect of adsorbent dosage on the adsorption of tolueneby WTRG $(\mathrm{pH} 8.5$; initial concentration, $500 \mathrm{mg} / \mathrm{L}$; temperature, $\left.20^{\circ} \mathrm{C}\right)$.

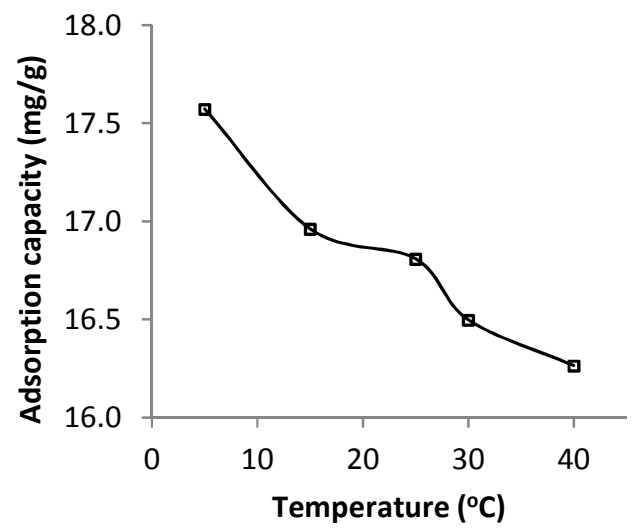

Fig. 4: Effect of temperature on the adsorption of toluene by WTRG ( $\mathrm{pH} 8.5$; WTRG dose $2 \mathrm{~g}$; initial concentration, $500 \mathrm{mg} / \mathrm{L}$ 


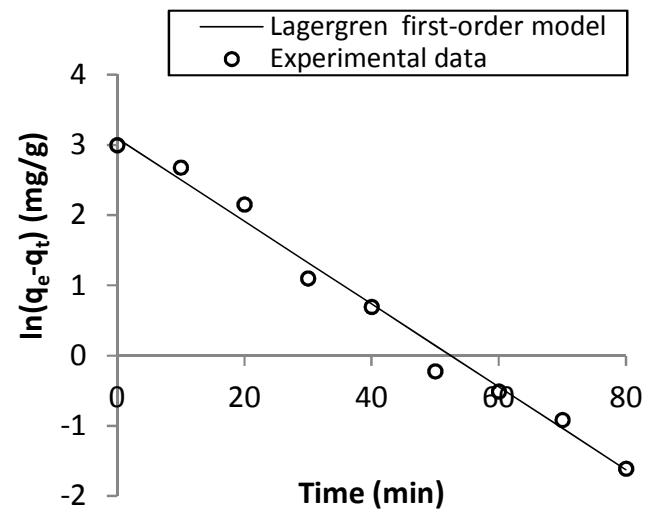

Fig. 5: Lagergren first-order kinetic model fitted

to the batch adsorption data obtained for toluene adsorption onto WTRG.

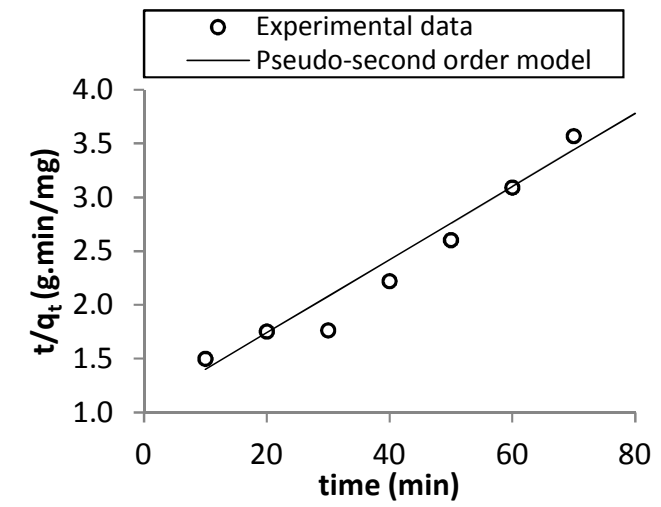

Fig. 6: Pseudo second order kinetic model fitted to the batch adsorption data obtained for toluene adsorption onto WTRG

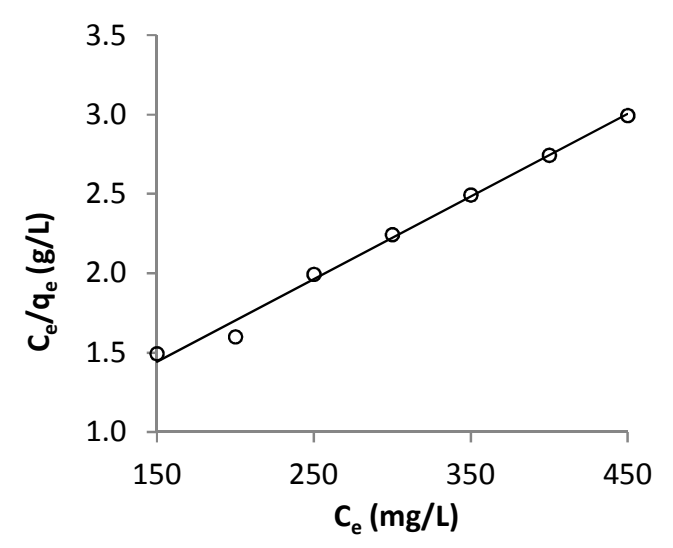

Fig. 8: Langmuir isotherm fitted to the batch adsorption data obtained for toluene adsorption onto WTRG

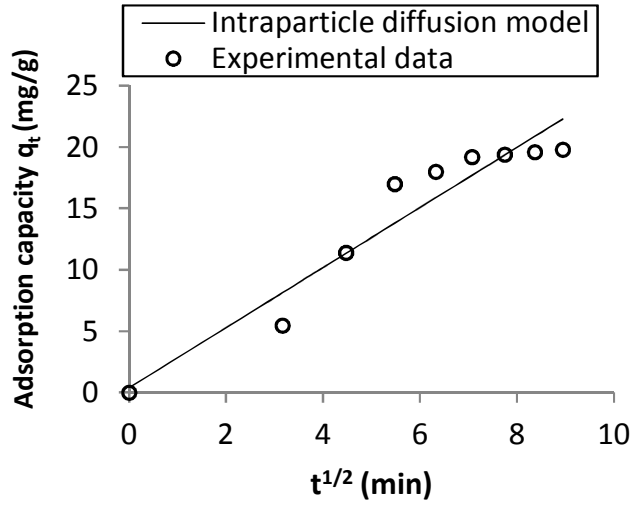

Fig. 7: Intra particle diffusion model fitted to the batch adsorption data obtained for toluene adsorption onto WTRG 


\section{REFERENCES}

Aisien, FA; Hymore, FK; Ebewele, RO (2006). Application of Ground Scrap Tyre Rubbers in Asphalt Concrete Pavement. Indian Journal of Engineering and Materials Science, 13: 333-338.

Aisien, FA; Amenaghawon, NA; Akhidenor, SA (2013). Adsorption of Ethylbenzene from Aqueous Solution using Recycled Rubber from Scrap Tyre. Journal of Scientific Research and Reports, 2(2): 497-512.

Ajay, KJ; Vinod, GK; Shubhi, J; Suhas (2004). Removal of Chlorophenol using industrial wastes. Environmental Science and Technology, 38: 1195 1200 .

Alam, MJB; Chowdhuary, RK; Hasan, MM; Huda, A; Sobhan, S (2006). Study and simulation of competitive sorption of 2,4,-D and phenol on waste tire rubber granules. ARPN Journal of Engineering and Applied Sciences, 1(3): 45.

Alamo-Nole, LA; Roman, F; Perales-Perez, O (2006). Sorption of ethyl benzene, toluene and xylene onto crumb rubber from aqueous solutions. MSc Thesis, University of Puerto-Rico.

Annadurai, G; Rajesh, BS; Mahesh, KPO; Murugesan, T (2000). Adsorption and Biodegradation of Phenol by Chitosan-Immobilised Pseudomonas Putida. Bioprocess Engineering. 22: 493-501.

Cunliffe, AM; Williams, PT (1998). Composition of oils derived from the batch pyrolysis of tyres, Journal of Analytical and Applied Pyrolysis. 44(2): 131-152.

González, JF; Encinar, JM; Canito, JL; Rodríguez, JJ (2001). Pyrolysis of automobile tyre waste. Influence of operating variables and kinetics study, Journal of Analytical and Applied Pyrolysis, 58: 667-683.

Gunasekara, AS; Donovan, JA; Xing, B (2000). Ground discarded tires remove naphthalene, toluene, and mercury from water, Chemosphere, $41 ; 1155-1160$.

Jadhav, DN; Vangara, AK (2004). Removal of Phenol from Wastewater using Sawdust and Sawdust Carbon. Indian Journal of Chemical Technology. 11; 35-45.
Kim, J; Park, J; Edil, T (1997). Sorption of Organic Compounds in the Aqueous Phase onto Tire Rubber. Journal of Environmental Engineering, 123(9); 827835.

Lee, JM; Lee, JS; Kim, JR; Kim, SD (1995). Pyrolysis of waste tires with partial oxidation in a fluidized-bed reactor, Energy, 20(10); 969-976.

Liu, HS; Mead, JL; Stacer, RG (1998). Environmental impact of recycled rubber in light fill applications: summary \& evaluation of existing literature. Chelsea Center for Recycling and Economic Development, University of Massachusetts Lowell, Technical Report \#2.

Mahvi, AH; Maleki, A; Eslami, A (2004). Potential of Rice Husk Ash for Phenol Removal in Aqueous Systems. American Journal of Applied Sciences, 1(4); 321-326.

Mousavi, HZ; Hosseynefar, A;Jaheed, V; Dehghani, SA (2010). Removal of Lead from Aqueous Solution using Waste Tyre Rubber Ash as Adsorbent. Brazillian Journal of Chemical Engineering. 27(1): 79-87.

Nagda, GK; Diwan, AM; Ghole, VS (2007). Potential of tendu leaf refuse for phenol removal in aqueous system, Applied Ecology and Environmental Research, Vol. 5(2); 1-9.

Rao, M; Bhole, AG (2000). Removal of Chromium using low cost adsorbents. Journal of American Environmental Microbiology, 27; 291-296.

Smith, CC; Anderson, WF; Freewood, RJ (2001). Evaluation of shredded tyre chips as sorption media for passive treatment walls. Eng. Geol. 60; 253-261.

Zafar, MN; Nadeem, R; Hanif, MA (2007). Biosorption of Nickel from Protonated Rice Bran. Journal of Hazardous Materials. 143; 478-485. 